\title{
MANAGING EVOLUTION IN TELECOMMUNICATION SYSTEMS
}

\author{
G.Koutsoukos $^{1}$, J.Gouveia ${ }^{1}$, L.Andrade ${ }^{1,2}$, J.L.Fiadeiro ${ }^{2,3}$ \\ 1 Oblog Software S.A. \\ Alameda Antonio Sérgio 7, 2795 Linda-a-Velha, PORTUGAL \\ \{gkoutsoukos.jgouveia,landrade\} @ oblog.pt \\ ${ }^{2}$ ATX Software S.A. \\ Alameda Antonio Sérgio 7, 2795 Linda-a-Velha, PORTUGAL \\ 3 Department of Informatics \\ Faculty of Sciences, University of Lisbon, Campo Grande, 1700 Lisboa, PORTUGAL \\ jose@fiadeiro.org
}

\begin{abstract}
Recent advances in telecommunication technology, including wireless networks and the Internet, along with the competition of network operators for offering advanced and different services, are putting increasing pressure for building telecommunication software systems that are adaptive to new requirements and easily reconfigurable, even in run time. We propose a new modelling primitive coordination contract - that we have developed and applied to other applications domains, as a means to provide an effective solution to this problem. We briefly describe coordination contracts and discuss how they can support the evolution of the specifications of the Wireless Application Protocol (WAP) Datagram layer.
\end{abstract}

Keywords: Component-based frameworks, Coordination, Evolution, Telecommunication Systems, Object-Oriented design, Reconfigurability, Scalability, Wireless Application Protocols

\section{INTRODUCTION}

Technology and system requirements in the telecommunications domain are changing very rapidly. Over the previous years, since the transition from ana$\log$ to digital communications, and from wired to wireless networks, different standards and solutions have been adopted, implemented and modified, often to deal with new and different business requirements. Today, more and more, telecommunication network operators strive to provide new advanced services in an attractive and usable way. However, time-to-market is a business decision that can be severely conditioned by the capacity of systems to ac- 
commodate changes quickly and with minimum impact on the services already implemented. This challenge is often difficult to be met by hardware-based systems because hardware cannot be easily modified and integrated.

On the other hand, thanks to the explosive growth of the Internet and the emergence of wireless data technologies, we are witnessing a major shift from hardware to software-based systems in this sector. This is because more and more applications must process data and information, a task that is easier to be performed on software. Therefore, it is not surprising that, due to their popularity in more traditional software application domains, object-oriented development techniques are becoming a standard in the telecommunications software industry. However, for reasons we put forward in [1], it is now widely accepted that, although $\mathrm{OO}$ techniques such as inheritance and clientship make it easier to build systems, their support for evolution in general, and the ability of systems to exhibit the agility required by the volatility of business domains in particular, is quite limited. Yet, the ability to change is now much more important than the ability to create systems in the first place. Change has become a first-class design goal that requires functional and technical architectures whose components can be added, replaced and reconfigured dynamically.

In this paper, we argue that the modelling primitive - coordination contract that we developed for superposing coordination mechanisms over existing components [1] can be applied to telecommunication systems in order to achieve increased flexibility and agility in reacting to change. By borrowing concepts and techniques from Reconfigurable Distributed Systems and Software Architectures, coordination contracts provide the ability for interactions between objects to be modelled as first-class entities and for changes that require a reconfiguration of such interactions to be performed without having to change the objects involved. Through an example related to the modelling of the Wireless Application Protocols, we discuss how coordination contracts can support such forms of evolution.

\section{COORDINATION CONTRACTS}

In general terms, a coordination contract is a connection that is established between a group of objects (participants), where rules and constraints are superposed on the behaviour of the participants, which determines a specific form of interaction. The way such an interaction is established between the partners is more powerful than what can be achieved within $\mathrm{OO}$ languages because it relies on the mechanism of superposition as developed for parallel and distributed system design [3]. When a call is made from a client object to a supplier object, the contract "intercepts" the call and superposes whatever forms of behaviour it prescribes. In order to provide the required levels of pluggability, neither the client, nor any other object in the system, needs to know what kind of coordina- 
tion is being superposed. To enable that, a contract design pattern, presented in [2], allows coordination contracts to be superposed on given objects in a system to coordinate their behaviour without having to modify the way the objects are implemented (black box view). In general terms, a coordination contract is defined as follows:

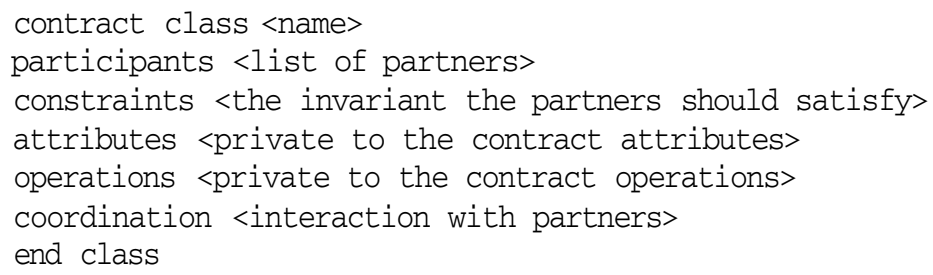

where each interaction under a coordination rule is of the form:

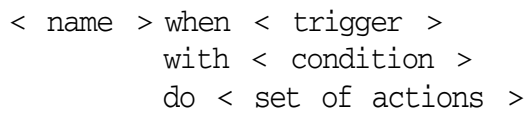

The condition under "when" establishes the trigger of the interaction. The trigger can be a condition on the state of the participants, a request for a particular service, or an event on one of the participants. The "do" clause identifies the reactions to be performed, usually in terms of actions of the partners and some of the contract's own actions. When the trigger corresponds to a request for an operation, three types of actions may be superposed on the execution of the operation: actions to be performed before the operation, a replace action which is performed instead of the operation (alternative) and actions that are performed after the operation. The "with" clause puts further constraints on the execution of the actions involved in the interaction. If any condition under the "with" clause is not satisfied none of these actions is executed. More details and references to other papers on the semantics and applications of coordination contracts can be found in [1,2].

\section{THE WAP DATAGRAM PROTOCOL}

The Wireless Application Protocol (WAP) is the latest attempt of the telecommunications industry to specify an application framework and network protocols for wireless devices with the main objective of bringing Internet content and advanced data services to digital cellular phones and other wireless terminals. A detailed description of the WAP architecture is presented in [4].

WAP layers are designed to operate over a variety of different bearer services supported by the various network types, i.e the data transport mechanisms used to carry data between two devices. For instance, WAP layers can operate 
over services such as GSM GPRS, CDMA CSD and so on. This functionality is accomplished in the layer referred to as the Wireless Datagram Protocol (WDP) [5]. WDP provides a common interface to the upper layers (Security, Session, and Application) of the protocol stack so that they are able to function independently of the underlying wireless network. This is achieved by adapting the transport layer to specific features of the underlying bearer. Therefore, the WAP layers architecture can, in fact, be considered as a 3 layered architecture of the upper layers, the underlying bearers and their interface (WDP).

In general terms, WDP has to perform 3 tasks: port addressing by assigning port numbers (identify the higher layer entity above WDP), segmentation of datagrams and re-assembly of packets and error reporting. Discussing the way WDP performs these tasks is out of the scope of this paper. The reader can consult [5] for more details. However, it is clear that the list of supported bearers will change over time with new bearer types and services being added as the wireless market evolves (projection made by WapForum in [4], pg 17). Moreover, specifications keep changing in order to improve the protocol, making relevant modifications to the implementation of the interface level (WDP) needed in order to continue offering transparent services to the upper layers of the WAP stack. Therefore, WDP must be flexible enough to accommodate the changes in the underlying level quickly and with minimum impact on the services already implemented.

\section{COORDINATING WDP COMPONENTS}

We will now discuss how the flexibility required on the WDP can be achieved using a contract based development methodology. As far as the evolution of bearers is concerned, a generic architecture of our proposal is shown below.

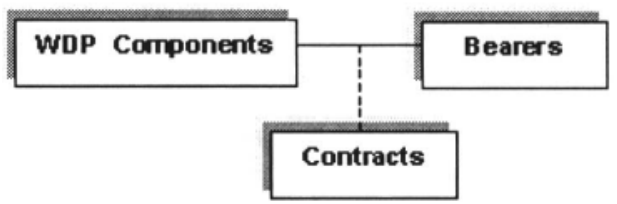

The WDP components correspond to the parts of the WDP layer that are identical for all bearer services supported by WDP. This means that they are computationally identical. However, their conditions for execution are different according to the underlying bearer. The WDP components can be implemented as "black boxes". It is the responsibility of the contracts to coordinate the behaviour of such components according to the specific requirements of a bearer service. When a new bearer (type or service) is to be added to the ones already supported by WAP, new contracts will be added to the system to support that bearer. As a result, the already implemented WDP components will remain un- 
changed, thus allowing support for the evolution of requirements and achieving software reuse.

Consider, for instance, the segmentation case. As already stated, WDP has to provide for segmentation and re-assembly of datagrams in a bearer dependent way. A datagram is a unit of information that consists of header fields and data fields. However, from the segmentation point of view, a datagram can be considered as a sequence of bits that is split into a number of packets being transmitted over the network. From the evolution point of view, the issue in segmentation is that the resulting packets must be of a size and format consistent with the underlying network service.

In a conventional design in which segmentation is implemented in different components in a bearer dependent way, the required evolution would be difficult to be achieved in a compositional way. However, contracts provide a very flexible solution to the problem. Consider a design in which a class Segmentation d9efines an operation Segment(Datagram) to perform the segmentation of a datagram into a number of packets. The Segmentation class and Segment are defined in such a way that they provide the necessary computational functionality that is common for all or some bearer types or services. All bearer specific features of segmentation, such as packet size, encoding of packets and so on are modelled in contracts. Each contract corresponds to a bearer service and is responsible for coordinating the segmentation operation according to the underlying bearer requirements. For instance, GSM_Service_Segmentation below could be the definition of a contract that is superposed on the Segmentation operation in order to support a GSM bearer service. The contract sets the maximum packet size for segmentation to be equal to the size required by the GSM Service. Moreover, it defines some operations for encoding the packet headers according to the particular GSM Service requirements. Additional operations or actions may be required based on more "low-level" design decisions.

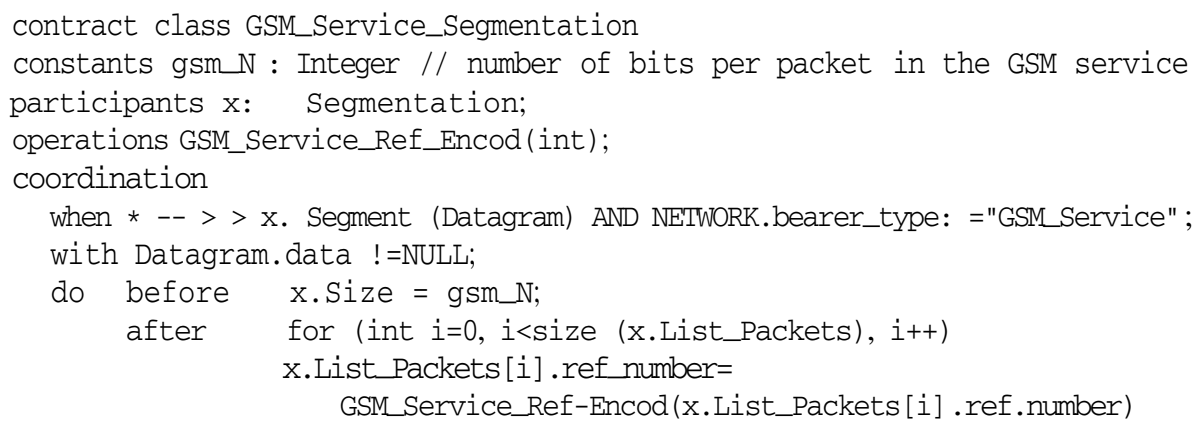

end contract

When a new service is added to the protocol, a new contract will be added to the system in a "plug and play" mode to support that service. This allows for 
the Segmentation component already defined and all client components using Segmentation to remain unchanged. It is also important to note that the condition specified by the keyword AND under the "when" clause checks whether the network service is the correct one. This is accomplished by using a NETWORK global component, defined only for illustrative purposes. If the condition is false, it may be possible for another contract, imposed on Segmentation and concerning another network type, to be executed. In that way, support for dynamic network reconfiguration may be achieved. This is an application of contracts that we intend to further investigate in the future. Finally, it should be mentioned that similar designs exist for the other operations of WDP as well as for telecommunications systems in general, but due to space limitations are not presented in this paper.

\section{CONCLUDING REMARKS}

The telecommunications sector is being governed by the expeditious growth of two networking technologies: the wireless data and the Internet. This growth has fuelled the creation of new and exciting information services and resulted in a major shift from hardware to software as far as the implementation of telecommunication systems is concerned. However, under this increasing pressure for new sophisticated services, and with the frequent adoption of new standards, software development in telecommunications cannot rely solely on traditional OO development techniques such as inheritance and clientship. We believe that the only hope for telecommunication organisations to be able to face the challenges of the fast market and technology evolution is to follow the emerging trend in software analysis and design based on predefined frameworks of skeletal applications, components, and design patterns that can be easily customized and integrated.

In this paper, we proposed the contract-based development methodology described in [1] as a discipline that can be applied when developing telecommunications systems in order to support the evolution of system requirements and achieve software reuse. We supported our view by briefly discussing how contracts can provide a compositional structuring mechanism with respect to changes occurring due to the evolution of specifications of the Wireless Application Protocol Datagram Layer. Based on our experience in applying the contract-based methodology in other domains, we believe that by adopting coordination contracts when building telecommunications systems increased levels of flexibility and reuse will be achieved.

\section{Acknowledgments}

Some of the ideas presented in this paper were developed as part of the first author's MSc thesis at King's College, University of London, September 2000, 
who would like to thank Prof. Tom Maibaum for his valuable guidance and comments.

\section{References}

[1] L.F.Andrade and J.L.Fiadeiro, "Coordination Technologies for Managing Information System Evolution”, in Proc. CAISE'01, A.Geppert (ed), LNCS, Springer-Verlag 2001.

[2] J.Gouveia, G.Koutsoukos, L.Andrade and J.L.Fiadeiro, "Tool Support for CoordinationBased Software Evolution", in Technology of Object-Oriented Languages and SystemsTOOLS 38, W.Pree (ed), IEEE Computer Society Press 2001, 184-196.

[3] S.Katz, "A Superimposition Control Construct for Distributed Systems", in ACM TOPLAS 15(2), 1993, 337-356.

[4] The WapForum. "The WAP Architecture Specification", Version 30th April 1998 http://www.wapforum.org/what/technical.htm.

[5] The WapForum. "The WAP Wireless Datagram Protocol Specification", Version 19th Feb 2000, http://www.wapforum.org/what/technical.htm. 